\title{
Clinical efficacy of marbofloxacin in dogs and cats diagnosed with lower urinary tract disorders")
}

\author{
BANU DOKUZEYLÜL, BARAN ÇELIK*, BELGI DIREN SIĞIRCI*, \\ BEREN BAŞARAN KAHRAMAN*, SINEM ÜLGEN SAKA, \\ ABDULLAH KAYAR, SEYYAL AK*, M. ERMAN OR \\ Department of Internal Medicine, *Department of Microbiology, Faculty of Veterinary Medicine, \\ Istanbul University-Cerrahpaşa, 34320, Avcılar-Istanbul, Turkey
}

Dokuzeylül B., Çelik B., Diren Sığırcı B., Başaran Kahraman B., Ülgen Saka S., Kayar A., Ak S., Or M. E. Clinical efficacy of marbofloxacin in dogs and cats diagnosed with lower urinary tract disorders

Summary

Marbofloxacin is one of the fluoroquinolones developed exclusively for veterinary medicine. The primary aim of the present study is to identify and assess evidence for marbofloxacin's clinical efficacy in the treatment of urinary tract infections in small animal practice. The study included 118 dogs and 123 cats that were referred to the Internal Medicine Department with lower urinary tract symptoms. We excluded animals that had received antimicrobial or anti-inflammatory therapy in the previous 15 days. Diagnosis was confirmed by clinical findings, urinalysis, and imaging. Rectal temperature, appetite, urinary signs, and abdominal pain were monitored during two visits (day 0 and 14). The timetable depended on the diagnosis of the following: two urinalyses, two bacterial examinations, and antibacterial susceptibility testing were performed for each case. Bacterial UTI were confirmed in 36 dogs and 28 cats. Urine samples were collected on day 0 , which demonstrated the presence of various bacteria, with a marked predominance of $P$. mirabilis and coagulase-positive Staphylococci in canine and feline urine samples, respectively. Antimicrobial susceptibility test results revealed $25(65.7 \%)$ of dog isolates and $24(85.7 \%)$ of cat isolates were susceptible to marbofloxacin. Treatment of UTIs is generally challenging for the small animal practitioner. Because of the need for long-term antimicrobials, bacterial culture and susceptibility tests are especially important for successful treatment. Marbofloxacin can be part of an effective treatment of UTIs in dogs and cats.

Keywords: antimicrobials, cat, dog, marbofloxacin, urinary tract infection

Urinary tract infections (UTI) are the microbial colonization of any part of the urinary tract. UTIs are opportunistic infections and generally occur when the host's defences are compromised and a virulent organism adheres, multiplies, and persists in a portion of the urinary tract (9). Treatment should include appropriate antimicrobials to eliminate the underlying abnormality and ensure recovery (4).

UTIs are one of the leading reasons for prescribing antibiotics in small animal practice. Recommended treatment time for canine and feline UTIs is relatively longer than humans from 4-7 days (in uncomplicated cases) and up to 28 days (in complicated cases) (9). Antimicrobial therapy is generally recommended for confirmed UTIs and treatment can help reduce the severity of renal injury due to microbial inflammatory damage (5).

${ }^{1)}$ This work was supported by Scientific Research Projects Coordination Unit of Istanbul University. Project number is BYP-2016-20321. The authors are thankful to Novakim Pharmaceuticals Company for the marbofloxacin cartridge discs.
Diagnosing a bacterial UTI requires a positive urine culture, isolation and identification of the organism and determination of the number of bacteria (colony-forming units per unit volume). Other tests may also be used in the diagnosis such as urine analyses, complete blood counts (CBC), chemistry profiles, rectal examinations, tests for feline leukemia virus and feline immunodeficiency virus, thyroid function tests, dexamethasone suppression tests, adrenocorticotropic hormone stimulation tests, abdominal radiographs, abdominal ultrasounds, contrast radiology, cystoscopy with bladder wall culture tests and prostatic washes (14).

Marbofloxacin (Marbocyl ${ }^{\circledR}$, Vétoquinol, United Kingdom) is a synthetic, broad- spectrum antibiotic of third-generation fluoroquinolones, which were exclusively developed for veterinary practice $(15,21)$. Marbofloxacin is recommended for treating respiratory infections, skin and soft tissue infections, genito-urinary tract infections, and prostatitis $(8,10)$. It has been ap- 
proved for use in dogs and cats at a dosage of 2-4 mg/ $\mathrm{kg}$ of body weight since 2001 (16). After oral administration, more than $70 \%$ is excreted in urine (15), so it is recommended for use in animals susceptible to UTIs (15). It has potent bactericidal activity against aerobic Gram-negative and positive organisms, mycobacteria, and rickettsia. It has a high clinical efficacy against many of the principal pathogens encountered in UTIs. However, fluoroquinolone resistance in staphylococci and enterococci is an emerging problem (21). Like other fluoroquinolones, marbofloxacin is contraindicated in immature animals younger than one year of age (16).

The bacteria that usually cause UTIs are similar in dogs and cats. Escherichia coli is the most common Gram-negative organism followed by Gram-positive cocci, other Gram-negative bacilli (Proteus spp., Klebsiella spp., Pasteurella spp., Pseudomonas spp.), Corynebacterium spp., and some rarely reported genera such as Mycoplasma spp. (14). The coagulase-negative strain Staphylococcus felis is the most common Grampositive cocci that causes lower UTIs in cats (7). The prevalence of lower UTIs is much lower in cats than in $\operatorname{dogs}(11,12)$.

The aim of the study is to identify and assess the evidence for the clinical efficacy of marbofloxacin in the treatment of lower UTIs in small animal practice.

\section{Material and methods}

Animals, in total 118 dogs and 123 cats of different breeds, weights and sexes, were referred with clinical signs consistent with UTIs (such as stranguria, hematuria, pollakiuria, inappropriate urination, excessive licking of the genital area, and frequent or prolonged attempts to urinate). They were referred to the study from the ambulatory clinics of the Department of Internal Medicine.

Exclusion criteria. Animals were excluded from the study if they had received antimicrobial or anti-inflammatory treatment within the previous 15 days. Animals under one year of age were excluded because of the side effects of fluoroquinolones on articular cartilage in growing animals (4). The clinical trial design considered all ethical aspects.

Pre-treatment evaluation. At the pre-treatment examination, patient information and general history were collected and physical examinations were performed that assessed rectal temperature, general health status, appetite, urinary signs, defecation disorders, and abdominal pain. Specific clinical signs that could indicate UTIs were documented for all patients. All urine samples were obtained by cystocentesis for laboratory examination. Strip tests and urine sediment examinations were performed. Urine specific gravity was measured with a veterinary refractometer. Complete urine analyses, qualitative bacterial examinations and susceptibility tests were performed. Radiographies and/or ultrasonographies of the abdomen were performed to determine the presence of significant abnormalities such as tumours, uroliths in the urinary tract, or anatomical defects. Marbofloxacin was prescribed for animals whose test results were confirmed by antimicrobial susceptibility tests. Marbofloxacin tablets were given $2 \mathrm{mg} / \mathrm{kg}$ orally SID for 10 days to animals diagnosed with lower UTIs. Dogs with prostatitis and/or persistent urinary tract infections received marbofloxacin at the same dosage for up to 28 days.

Bacteriological examination. All urine samples were tested in the laboratory of the Microbiology Department of Faculty of Veterinary Medicine. Bacterial infection was confirmed with cultures taken at the first inspection on day 0 . A bacterial count of more than $10^{3} \mathrm{cfu} / \mathrm{ml}$ was considered diagnostic of a UTI (17). Aerobic and anaerobic bacterial species were identified using standard methods based on morphology, Gram stain, growth characteristics, standard biochemical tests, or commercial identification kits (API systems, BioMérieux, SA, Marcy I'Etolie, France) (18, 19). Cultures with no growth after 7 days were interpreted as negative.

Antimicrobial susceptibility tests. Antimicrobial susceptibility tests were performed according to the Kirby-Bauer Method recommended by the Clinical Laboratory Standards Institute (CLSI) to select the suitable antimicrobial agent for treatment (1). The inhibition zone around the disk (Marbocyl, $5 \mu \mathrm{g}$ ) was measured. Inhibition zones of $18 \geq \mathrm{mm}$ were considered susceptible, $14-17 \mathrm{~mm}$ were considered intermediate, and $14 \leq$ were considered resistant $(1,13)$.

Post-treatment evaluation. Post-treatment evaluations were conducted for all patients to assess the clinical response to marbofloxacin (after 10-28 days, but generally on the $14^{\text {th }}$ day). The evaluation was based on the rates of clinical cure, bacteriological cure and relapse. The clinical assessment took into account the disappearance of all general and UTI symptoms recorded on day 0 . Bacteriological cures were confirmed when the levels of organisms isolated on day 0 were below the positive threshold on day 14. Cases with levels above this threshold were considered relapses.

Statistical analysis. The results were analyzed with the program SPSS 14.0. The Mann-Whitney U test was used to compare the number of leukocytes in each field in the pre and post-treatment urine samples. Differences were considered significant at $\mathrm{p}<0.05$.

\section{Results and discussion}

The study included 118 dogs (49\%) and 123 cats (51\%). The most common dog breeds were mixed (24\%), Golden Retriever (14\%) and German Shepherd (11\%). In dogs, we diagnosed urolithiasis, UTI, haemorrhagic cystitis, urethritis, prostatic diseases, urinary incontinence, urinary calculi, transitional cell carcinoma (TCC). UTI (31\%), urolithiasis (28\%), haemorrhagic cystitis $(19 \%)$ were the most frequently seen disorders in dogs.

The most common cat breeds were mixed (73\%), Persian (15\%) and Siamese (6\%). In cats, we diagnosed UTI, hemorrhagic cystitis, urolithiasis, urethral plaque, idiopathic cystitis, urethritis, polypoid cystitis and TCC. The most common lower urinary tract diseases were urolithiasis (33\%), UTI (22\%) and idiopathic cystitis $(20 \%)$ (Fig. 1). Hematology and blood serum biochemistry test results revealed no significant alterations, and all post-treatment values remained within the normal ranges.

The most common clinical signs of UTIs in dogs was pollakiuria, which was present $47 \%$ of the dogs. 


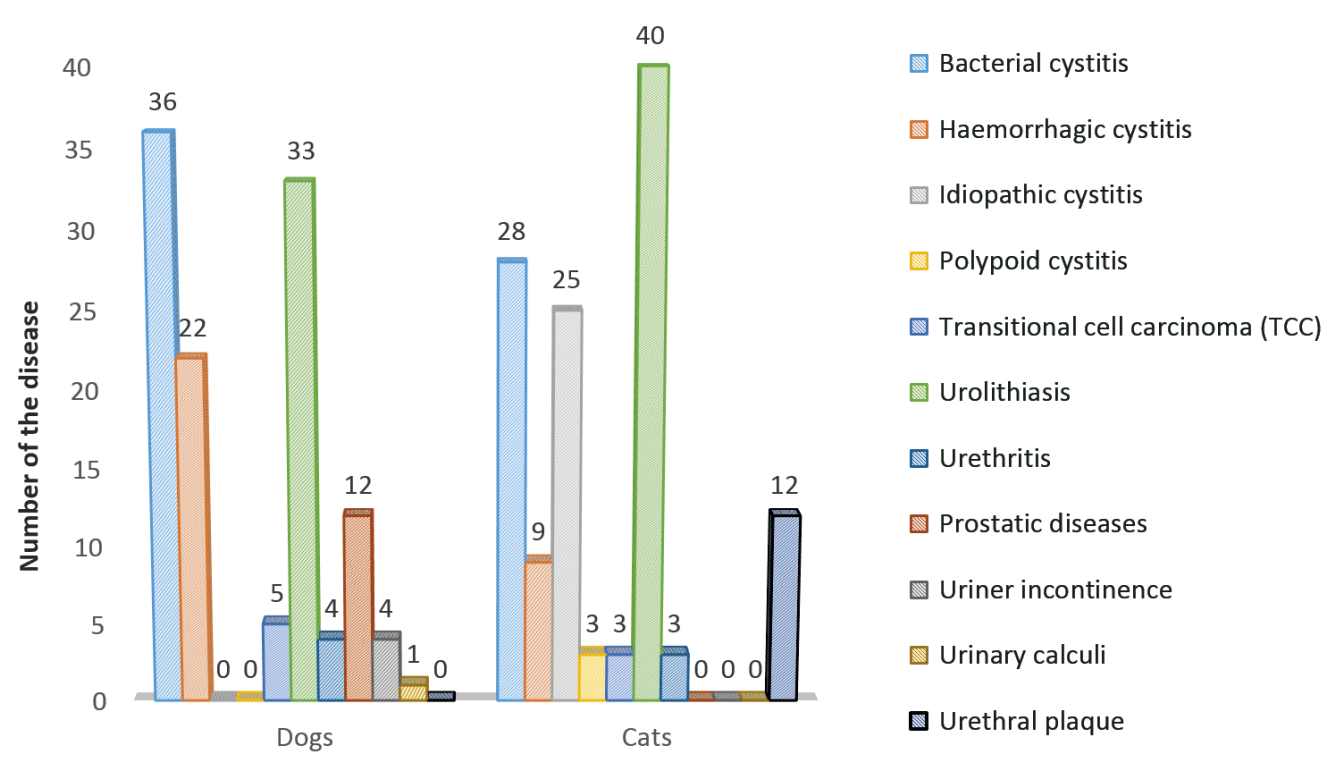

Fig. 1. Distribution of lower urinary tract diseases in cats and dogs
The most common isolates were $E$. $\operatorname{coli}(\mathrm{n}=15 / 28$ Gram-negative isolates) and coagulase-positive staphylococci ( $\mathrm{n}=16 / 43$ Grampositive isolates). There were six UTIs involving two bacterial species, E. coli in combination with $P$. mirabilis or $P$. aeruginosa. One specimen included E. faecalis in combination with $S$. intermedius and two specimens included S. canis-in combination with A. baumannii or $S$. intermedius. Antimicrobial susceptibility tests showed that 25 $(65.7 \%)$ of dog isolates and $24(85.7 \%)$ of cat isolates

The most common clinical sign of UTIs in cats was stranguria, which was present more than $59 \%$ of the cats (unpublished data).

Bacterial UTI were confirmed in 36 dogs and 28 cats. Urine samples from day 0 had various bacteria, with a marked predominance of $P$. mirabilis and coagulase positive staphylococci in canine and feline urine samples, respectively (Fig. 2 and 3).

were susceptible to marbofloxacin.

Fifteen bacteria species were resistant to marbofloxacin. In these cases, treatment was continued with other antimicrobials (Tab. 1). The clinical cure rate was $87.3 \%$. The bacteriological cure rate was $96 \%$ and there were no relapses.

Marbofloxacin has good activity against many Gram-negative bacilli and cocci including E. coli, P. aeruginosa, Enterobacter, Campylobacter,

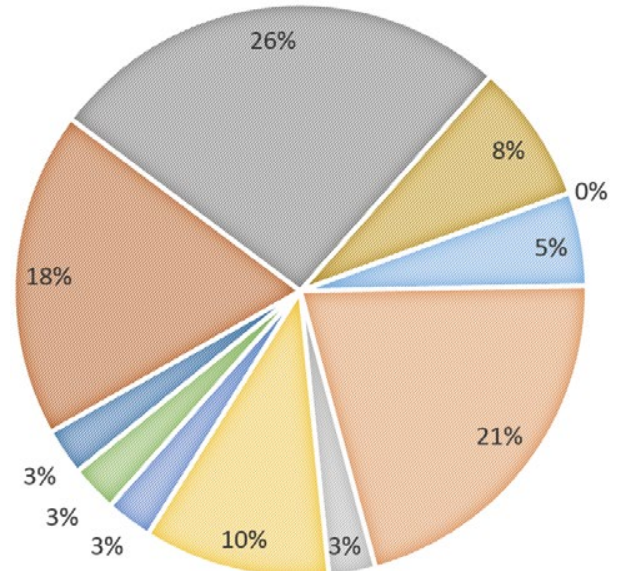

= Coagulase-negative staphylococci

= Coagulase-positive staphylococci

= A. baumannii

= Streprococci

- E. faecalis

$=$ A. viridans

- Micrococcus sp.

- E. coli

- P. mirabilis

" Other Gram-negative bacilli

Fig. 2. Isolates obtained from dogs diagnosed with lower UTIs

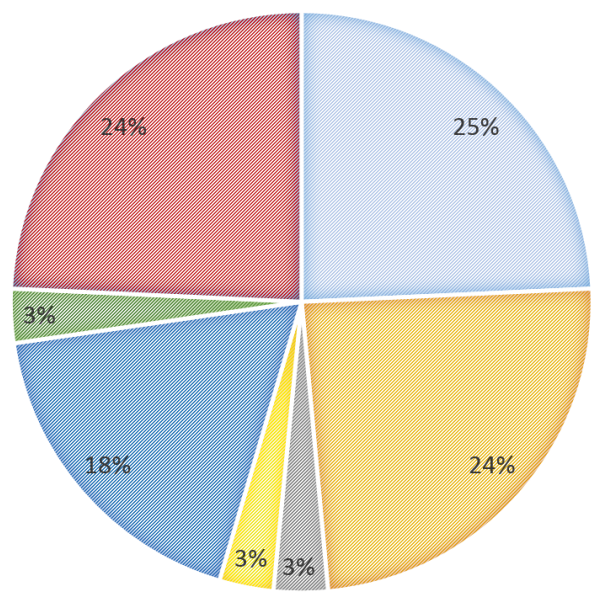

- Coagulase-positive staphylococci

Coagulase-negative staphylococci

E. avium

E. cloacea

E. faecalis

S. canis

E. coli

Fig. 3. Isolates obtained from cats diagnosed with lower UTIs
Proteus, Staphylococcus, Mycoplasma, and

Tab. 1. Susceptible antimicrobials for marbofloxacin resistant isolates

\begin{tabular}{|l|l|}
\hline \multicolumn{1}{|c|}{ Isolate } & \multicolumn{1}{c|}{ Susceptibility profile } \\
\hline P. aeruginosa & AMK; CAZ; CIP \\
\hline Str. canis & AMC; CRO; SXT \\
Str. bovis & AMC; CRO; STR; SXT \\
S. aureus & CIP; CAZ \\
\hline S. aureus & AMK; AMC; CFZ; CXM; CRO; CEF; SXT \\
S. intermedius & SXT; CHL \\
\hline S. intermedius & AMK; AMC;CRO; CFZ; CHL; CLR \\
\hline E. coli & GEN; PEN; AMP \\
\hline E. coli & AMC; SAM; SXT; TET \\
\hline P. mirabilis & CAZ \\
\hline E. avium & STR \\
A. baumanii & - \\
\hline S. epidermidis & SXT; CAZ; CRO;TET; CIP; AMK; AMC \\
\hline P. stutzeri & AMK; CXM; CFZ; CIP; SAM; SXT \\
\hline E. cloaca & AMK; AMC; CFZ; CXM; CRO; TET; SXT \\
\hline
\end{tabular}

Explanations: AMK - amikacin; AMC - amoxicillin-clavulanic acid; AMP - ampicillin; SAM - ampicillin-sulbactam; CFZ - cefazolin; CAZ - ceftazidime; CRO - ceftriaxone; CXM - cefuroxime; $\mathrm{CEF}$ - cephalotin; CHL - chloramphenicol; CIP - ciprofloxacin; CLR clarithromycin; GEN - gentamycin; PEN - penicillin; STR - streptomycin; TET - tetracyclin; SXT - trimethoprim-sulfamethoxazole 
Mycobacterium. The effect on the Streptococci species varies, so the drug is not recommended for treating this species. Fluoroquinolones generally have a weak effect on anaerobes. Resistance to fluoroquinolones mostly occurred due to mutations in Pseudomonas, Klebsiella pneumonia, Acinetobacter and Enterococci (20).

Cotard et al. (4) reported that marbofloxacin had bactericidal activity over a broad spectrum that covers the main pathogens involved in UTIs in small animal practice, particularly E. coli and most of the Enterobacteriaceae, Staphylococcus, and Pseudomonas species. In the current study, the clinical bacteriological cure rates showed that marbofloxacin was effective in treatment of UTIs. There were high concentrations of marbofloxacin in urine $(49.5 \mu \mathrm{g} / \mathrm{ml})$ after oral administration with a dosage of $2 \mathrm{mg} / \mathrm{kg} /$ day (13).

In treatment of UTIs, suitable drugs and doses must be assessed carefully, considering the animal's metabolic functions and susceptibility of the agent causing the UTI. Fluoroquinolones were not the first choice for uncomplicated UTIs, according to CLSI (3). Marbofloxacin could be and should be prescribed for complicated UTIs or unresponsive cases with previous treatment failure.

It is well known that misuse one of the fluoroquinolones (ciprofloxacin, enrofloxacin, or marbofloxacin) leads the others to become resistant (6). In the current study, when fluoroquinolone resistance was identified, the history of the individuals showed excessive use of fluoroquinolones, especially ciprofloxacin, in most cases (unpublished data).

In the current study, one of the Enterococci strains that was resistant to marbofloxacin and therapy was conducted with another antimicrobial agent. The two other Enterococci strains were susceptible to marbofloxacin, but it was not used for treatment because these strains have a high frequency of intrinsic resistance.

Acinetobacter baumannii is an emerging Gramnegative multidrug-resistant (MDR) nosocomial pathogen. A. baumannii was previously treated with Fluoroquinolones to which it has recently become resistant (2). In the current study, a multi-drug resistant A. baumannii strain was isolated from a canine urine sample. This finding is very important and could be a serious public health risk, since transmission is quite possible through a pet's close contact with its owners and neighbors.

Olin and Bartges (14) reported that a single bacterial pathogen was isolated from approximately $75 \%$ infections, $20 \%$ of UTIs were caused by two co-infecting species, and approximately $5 \%$ are caused by three species. In the current study, a single bacterial pathogen was isolated from approximately $92.1 \%$ of the infections, $7.8 \%$ of UTIs were caused by two co-infecting species, and none were caused by three species. Six of the samples $(9.3 \%)$ contained two species. Urine samples were collected by cystocentesis to eliminate iatrogenic bacterial contamination as a confounding factor (11).
Treatment of UTIs is generally challenging for the small animal practitioner. Because long-term antimicrobial usage is necessary, bacterial cultures and susceptibility tests are very important factors for treatment success. Marbofloxacin usage can be effective in the treatment of UTIs in dogs and cats.

\section{References}

1. Apley M. B., Brown S. D., Gray J. T., Heine H., Hunter R. P., Mevius D. J., Papich M. G., Silley P., Zurenko G. E.: Performance standards for antimicrobial disk and dilution susceptibility tests for bacteria isolated from animals, approved standard-third edition. CLSI document M31-A3. Clinical and Laboratory Standards Institute, Wayne P. A., USA 2008.

2. Chopra S., Galande A.: A fluoroquinolone-resistant Acinetobacter baumannii without the quinolone resistance-determining region mutations. J. Antimicrob. Chemother. 2011, 66, 2668-2670.

3. CLSI document M02-A10, CLSI. 2009.

4. Cotard J., Gruet P., Pechereau D., Moreau P., Thomas E., Deleforge J.: Comparative study of marbofloxacin and amoxicillinclavulanic acid in the treatment of urinary tract infections in dogs. J. Small Anim. Pract. 1995, 36, 349-353.

5. Freitag T., Squires R. A., Schmid J., Elliott J., Rycroft A. N.: Antibiotic sensitivity profiles do not reliably distinguish relapsing or persisting infections from reinfections in cats with chronic renal failure and multiple diagnoses of Escherichia coli urinary tract infection. J. Vet. Intern. Med. 2006, 20, 245-249.

6. Gebru E., Choi M. J., Lee S. J., Damte D., Park S. C.: Mutant-prevention concentration and mechanism of resistance in clinical isolates and enrofloxacin/ marbofloxacin-selected mutants of Escherichia coli of canine origin. J. Med. Microbiol. 2011, 60, 1512-1522.

7. Gerber B., Boretti F. S., Kley S., Laluha P., Muller C. N., Sieber S., Unterer M., Wenger M., Fluckiger Glaus T., Reusch C. E.: Evaluation of clinical signs and causes of lower urinary tract disease in European cats. J. Small Anim. Pract. 2005, 46, 571-577.

8. Ihrke P. J., Papich M. G., Demanuelle T. C.: The use of fluoroquinolones in veterinary dermatology. Vet. Dermatol. 1999, 10, 193-204.

9. Jessen L. R., Sorensen T. M., Bjornvad C. R., Nielsen S. S., Guardabassi L. Effect of antibiotic treatment in canine and feline urinary tract infections: a systematic review. Vet. J. 2015, 203, 270-277.

10. Lefebvre H. P., Schneider M., Dupouy V., Laroute V., Costes G., Delesalle L., Toutain P. L.: Effect of experimental renal impairment on disposition of marbofloxacin and its metabolites in the dog. J. Vet. Pharmacol. Ther. 1998, 21, 453-461.

11. Litster A., Moss S., Platell J., Trott D. J.: Occult bacterial lower urinary tract infections in cats-urinalysis and culture findings. Vet. Microbiol. 2009, 136, 130-134.

12. Litster A., Thompson M., Moss S., Trott D.: Feline bacterial urinary tract infections: An update on an evolving clinical problem. Vet. J. 2011, 187, 18-22.

13. Marbofloxacin reference book:1999, Vetoquinol

14. Olin S. J., Bartges J. W.: Urinary tract infections: treatment/comparative therapeutics. Vet. Clin. North Am. Small Anim. Pract. 2015, 45, 721-746.

15. Pallo-Zimmerman L. M., Byron J. K., Graves T. K.: Fluoroquinolones: then and now. Compend. Contin. Educ. Vet. 2010, 32, E1-9.

16. Paradis M., Abbey L., Baker B., Coyne M., Hannigan M., Joffe D., Pukay B., Trettien A., Waisglass S., Wellington J.: Evaluation of the clinical efficacy of marbofloxacin (Zeniquin) tablets for the treatment of canine pyoderma: an open clinical trial. Vet. Dermatol. 2001, 12, 163-169.

17. Passmore C. A., Sherington J., Stegemann M. R.: Efficacy and safety of cefovecin for the treatment of urinary tract infections in cats. J. Small Anim. Pract. 2008, 49, 295-301.

18. Quinn P. J., Markey B. K., Leonard F. C., FitzPatrick E. S., Fanning S., Hartigan P.: Veterinary Microbiology and Microbial Disease. John Wiley \&Sons, first edition, 1994

19. Quinn P. J., Markey B. K., Leonard F. C., FitzPatrick E. S., Fanning S., Hartigan P.: Veterinary Microbiology and Microbial Disease. John Wiley\& Sons, fourth edition, 2002.

20. Tucak P.: Plumb's Veterinary Drug Handbook. DC Plumb, Wiley-Blackwell 2015, 1296.

21. Yohannes S., Awji E. G., Lee S. J., Park S. C.: Pharmacokinetics and pharmacokinetic/pharmacodynamic integration of marbofloxacin after intravenous and intramuscular administration in beagle dogs. Xenobiotica 2015, 45, 264-269.

Corresponding author: Banu Dokuzeylül, Assoc Prof, Istanbul UniversityCerrahpaşa, Faculty of Veterinary Medicine, Department of Internal Medicine, 34320, Avcılar, Istanbul, Turkey; e-mail: bdokuzeylul@gmail.com, b9eylul@istanbul.edu.tr 